\title{
Data Mining for Robust Business Intelligence Solutions
}

\author{
Jan Mrazek \\ Bank of Montreal, Global Information Technology, 4100 Gordon Baker Road, \\ Toronto, Ontario, M1W 3E8, Canada \\ jmrazek@sympatico.ca
}

Data Mining has quickly matured out of isolated, small scale, PC based, single algorithm techniques to robust analytical solutions which utilize a combination of various artificial intelligence algorithms, massively parallel technology, direct both-way access to relational databases and open systems with published Application Programming Interfaces. For an organization, this presents an opening of new opportunities, but it also generates a number of integration challenges.

This tutorial will introduce Data Mining from the perspective of a large organization. It will describe why Data Mining is essential for new business development and will present its enablers and drivers. Typical historically diversified organizational structure of Business Intelligence competency in a large organization will be introduced and the challenges posed to a large scale implementation of Data Mining will be portrayed.

Briefly, core technologies and techniques will also be introduced (Classification, Segmentation, Value Prediction, Basket Analysis, Time Series). Some hints on using Time greedy techniques will be given. Data Mining processes will be discussed in detail along with a brief genealogy of Data Mining technology and the criteria used for selecting the right technology. There will be a special focus on models for segmentation and cross-selling. These will be accompanied by brief case study demonstrations. The necessity of analyzing interaction of different business life cycles will be explained. A number of business applications of Data Mining will be listed. Special attention will be paid to the ability to determine account profitability on the lowest possible level of detail. A technically sophisticated profitability system will be introduced.

Data Mining will be presented in its integrity with other Business Intelligence solutions. The architecture of a large corporate Business Intelligence complex will be discussed from the technological as well as the business perspective. The delegates will be presented with the problem of the integration of Data Mining with the corporate Data Warehouse, Data Marts and OLAP. Challenges will be listed along with some feasible solutions. 
Data Warehouses are usually designed in 3rd normalized form or close to it. Data Marts use star or snowflake design (to support OLAP solutions). Neither one really suits Data Mining purposes. A design for Data Warehouse/Data Mart to satisfy all needs, including Data Mining will be discussed. In relation to Data Mining needs, some important data modeling techniques and modeling challenges will be explained. Examples are horizontal vs. vertical data structures, star and snowflake schemas, handling of slow changing dimensions, etc.

Data Mining starts with good data. Therefore special attention will be paid to data quality issues: insufficient data, outliers, missing values, data enrichment and data transformation. Data Mining techniques will be discussed from the perspective of requirements for data, its quality and its structure and type.

In a case study, an advanced concept for Behavior Oriented Segmentation will be shown. This will support the author's conviction that behavioral segmentation is more important than segmentation based on demographics and psychographics. It will be shown how these approaches complement each other. A link to customer profitability will be emphasized.

Challenges of mass deployment of Data Mining capabilities in an organization will be shown from the perspective of metadata management, decision timing and quality of results. Data Mining is a dangerous weapon, which may cause self-injury. Therefore some corporate safety rules will be introduced.

Finally, a vision of the near future of Data Mining and Data Mining Technology will be portrayed.

WEB References:

http://www.dw-institute.com/montreal.html

http://www.software.ibm.com/data/solutions/customer/montreal/montreal.htm http://www.cio.com/archive/051598_mining_content.html

http://www2.computerworld.com/home/print9497.nsf/All/SL42mont16896

http://www.bmo.com/

http://sites.netscape.net/drjanmrazek/ 